\title{
Smoke detection in video surveillance: a MoG model in the wavelet domain
}

\author{
Simone Calderara, Paolo Piccinini, and Rita Cucchiara \\ DII, University of Modena and Reggio Emilia, Italy \\ \{calderara.simone, piccinini. paolo, cucchiara.rita\}@unimore.it
}

\begin{abstract}
The paper presents a new fast and robust technique of smoke detection in video surveillance images. The approach aims at detecting the spring or the presence of smoke by analyzing color and texture features of moving objects, segmented with background subtraction. The proposal embodies some novelties: first the temporal behavior of the smoke is modeled by a Mixture of Gaussians (MoG ) of the energy variation in the wavelet domain. The MoG takes into account the image energy variation due to either external luminance changes or the smoke propagation. It allows a distinction to energy variation due to the presence of real moving objects such as people and vehicles. Second, this textural analysis is enriched by a color analysis based on the blending function. Third, a Bayesian model is defined where the texture and color features, detected at block level, contributes to model the likelihood while a global evaluation of the entire image models the prior probability contribution. The resulting approach is very flexible and can be adopted in conjunction to a whichever video surveillance system based on dynamic background model. Several tests on tens of different contexts, both outdoor and indoor prove its robustness and precision.
\end{abstract}

Key words: Smoke detection, Image processing, MoG, DWT

\section{Introduction}

Smoke detection in video-surveillance systems is still an open challenge for computer vision and pattern recognition communities. It concerns the definition of robust approaches to detect, as soon as possible, spring and fast propagation of smoke possibly due to explosions, fires or special environmental conditions. Smoke detection module can enrich standard video surveillance systems for both indoor and outdoor monitoring. These systems can replace standard smoke and fire sensors, which cannot be applied in large and open spaces. Moreover, detecting smoke by visual cues could allow fast and reactive alarms also in some specific situations, where smoke is growing in unconventional directions, so that the time-to-alarm of normal sensors could become unacceptable.

The video analysis tasks for smoke detection are not trivial due to the variability of shape, motion and texture patterns of smoke, which appearance is dependent on the luminance conditions, the background manifolds and colors of the scene. 
Since smoke modifies the visual cues of the background, typically background suppression techniques are adopted, followed by validation/classification tasks. The smoke identification becomes more challenging in presence of other moving objects and shadows and whenever the background is variable too.

The problem of smoke detection has been discussed in the past in some works where local features of pixels in the images or measures on the shape temporal variations are exploited. In an early work, Kopilovic et al. [1] took advantage of irregularities in motion due to non-rigidity of smoke. They computed optical flow field using two adjacent images, and then used the entropy of the motion directions distribution as key feature to differentiate smoke motion from nonsmoke motion. Similarly, motion was exploited in [2] where local motions from cluster analysis of points in a multidimensional temporal embedding space are extracted. The goal was to track local dynamic envelopes of pixels, and then use features of the velocity distribution histogram to discriminate between smoke and various natural phenomena such as clouds and wind-tossed trees that may cause such envelopes. In this work, the presence of other moving objects, typical of video-surveillance scenes, has not taken into account.

Recently, Chen, Yin et al. [3] present a smoke detection approach working on pixel-level classification after motion segmentation based on frame difference. Pixels can be initially classified as a smoke-pixel with a very simple chromaticitybased static decision rule; it is based on two thresholds in the color space assuming that smoke usually displays grayish colors. A Further dynamic decision rule is dependent on the spreading attributes of smoke: the ratio between the sums of circumferences of smoke regions segmented and the number of smoke-pixel extracted can give a measure of disorder in the segmented objects. Similarly other works evaluate the contours of the object that are candidate to be classified as smoke. In [5], smoke detection is based on four steps: background subtraction, flickering extraction, contour initialization, and contour classification using both heuristic and empirical knowledge about smoke. Background subtraction uses the Stauffer and Grimson algorithm [4]. Then a measure of flickering is provided. They state that flickering frequency of turbulent flame has shown experimentally to be around $10 \mathrm{~Hz}$ and it could be as low as 2 or $3 \mathrm{~Hz}$ for slowly-moving smoke. The temporal periodicity can be calculated using Fast Fourier Transform (FFT), Wavelet Transform or Mean Crossing Rate (MCR). They adopt the Mean Crossing Rate (MCR). Finally as in [3], a measure of the shape complexity given by the ratio between edge length and area is provided to achieve classification. Also in this work, only qualitative measure are provided.

An interesting and robust approach has been defined by Toreyin et. al. [6] and further improved in [7]. They use the Collins background subtraction method to extract moving objects [8]. Then as in previous work a flickering analysis and a measure of turbulence is provided by evaluating the edge and texture variation using the Wavelet Transform. In each block of the sub-image resulting after the wavelet decomposition the variation of energy is computed. The energy is given by the sum of the high-frequency components in the wavelet domain. Finally two thresholds are given to measure an acceptable energy variation. The 
dynamism of the variation is modeled with a simple three state Random Markov Model (RMM), trained with smoke and non-smoke pixels. Finally, an analysis of smoke shape complexity is provided as in [3] and [5], based on the distance between the contour points and the center of mass of the shape. This approach is quite robust in the given examples, but a precise evaluation of the different features contributions is not provided. However in our experiments, we observed that sometimes a strong shape variation and edge complexity of smoke regions cannot be visually revealed due to both camera field of view and wind direction. For this reason we avoid to use this feature.

Instead, we tested an higher discriminative power of wavelet with respect to Mean Crossing Rate, thus we adopt this transform for energy analysis. In our approach, moving objects are extracted with a standard background suppression technique and blobs subsequently classified as real objects or artifacts due to the smoke. The energy is computed using the coefficients of the Wavelet Transform; the RMM is substituted with a different statistical approach which takes into account the energy time-variability using a MoG computed with the online mixture model approach also used in [4]. In addition, the MOG classification is improved in a Bayesian model accounting the chromaticity variations by means of a blending function.

Differently from most of the previous approaches we do not make any assumptions both on the external conditions and on the field of view of the camera making our system flexible enough to be applied on different setups. Several tests have been carried out also taking videos form the Web. We evaluate the system performance measuring both the detection rate and the time to detect. The video used as test set are publicly available with annotation on the website http://imagelab.ing.unimore.it/visor. The paper is structured as follows. Section 2 describes the feature used for smoke detection and the adopted Bayesian Classifier. Finally Section 3 details all the system parameters and discuss the experiments carried out.

\section{Smoke detection for foreground object classification}

The proposed model evaluates the joint contribution coming from the graylevel image energy and color intensity attenuation to classify an object as possible smoke. We assume that when smoke grows and propagates in the scene its image energy is attenuated by the blurring effect of smoke diffusion.

We firstly detect possible candidate objects by means of a motion segmentation algorithm. When a new foreground object is detected we analyze its energy using the Wavelet Transform coefficients and evaluate its temporal evolution. The color properties of the object are analyzed accordingly to a smoke reference color model to detect if color changes in the scene are due to a natural variation or not. The input image is then divided in blocks of fixed sized and each block is evaluated separately. Finally a Bayesian approach detect whether a foreground object is smoke. 


\subsection{Energy analysis using the direct wavelet transform}

An efficient way to evaluate the energy variation of an intensity image is the discrete wavelet transform DWT [10].

The DWT is obtained convolving the image signal with several banks of filters obtaining a multiresolution decomposition of the image. Given the input image $I_{t}$ the decomposition produces four subimages, namely the compressed version of the original image $C_{t}$, the horizontal coefficient image $H_{t}$, the vertical coefficient image $V_{t}$ and the diagonal coefficient image $D_{t}$. An example decomposition is computed with the algorithm proposed in [10] is shown in Fig. 1

The energy is evaluated blockwise dividing the image in regular blocks of fixed
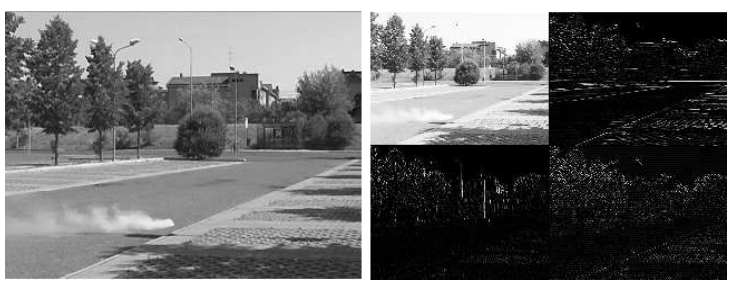

Fig. 1. Example of discrete wavelet transform. The leftmost image is the original image. The right image is the transformed one. The components are: top left compressed image $C_{t}$, top right horizontal coefficient image $H_{t}$, bottom left vertical coefficient image $V_{t}$ and bottom right diagonal coefficient image $D_{t}$.

size and summing up the squared contribution coming from each coefficient image:

$$
E\left(b_{k}, I_{t}\right)=\sum_{i, j \in b_{k}} V_{t}^{2}(i, j)+H_{t}^{2}(i, j)+D_{t}^{2}(i, j)
$$

where $b_{k}$ is the $k^{t h}$ block in the input image $I^{t}$.

The energy value of a specific block varies significantly over time in presence or absence of smoke, Fig. 2. When the smoke covers part of the scene the edges
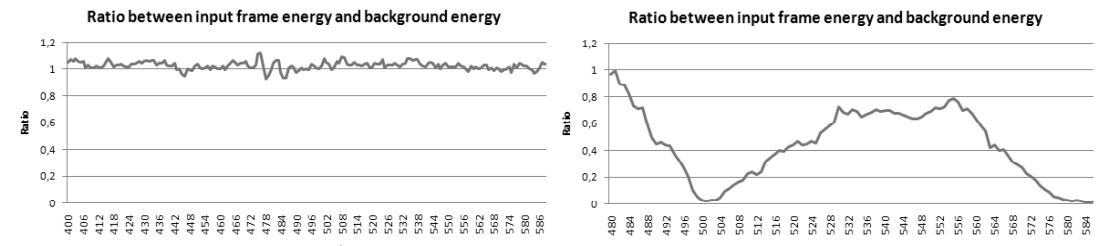

Fig. 2. Left figure: the energy ratio trend of a non smoke block. Right figure: the energy ratio trend of a smoke block. In presence of smoke the energy ratio is subjected to gradual drops in its value. 
are smoothed and the energy consequently lowered. This energy drop can be further emphasized computing the ratio $r\left(B_{k}\right)$ between the image energy of the current input frame and the one of the background model. The energy ratio has the advantage of normalizing the energy values and allowing a fair comparison between different scenes where the block energy itself can vary significantly. The ratio of the block $b_{k}$ is given by:

$$
r\left(b_{k}, I_{t}, B g_{t}\right)=\frac{E\left(b_{k}, B g_{t}\right)}{E\left(b_{k}, I_{t}\right)}
$$

where $B g_{t}$ is the background model up to time $t$ and $I_{t}$ is the input frame.

The analysis of the energy ratio is performed in two different context to account for both global and local energy drops.

Firstly the image energy variation is computed frame by frame to bias the detection using global information. Several clips containing a smoke events have been analyzed and the global energy ratio of the scene computed by sum the block energy. The Parzen window technique is adopted to build a non parametric distribution from global energy ratio values computed on several clips. The parzen window method is a kernel density estimator that computes a non parametric distribution from a set of iid samples $X=\left\{x_{i} \mid i=1 \ldots N\right\}$ of a random variable $x$. Adopting a specific kernel distribution the approximated pdf is computed summing the kernel for all the sampled values:

$$
\widehat{f}=\frac{1}{N h} \sum_{i=1}^{N} K\left(x-x_{i}\right)
$$

using a standard Gaussian kernel function $K=\frac{1}{2 \pi} e^{-\frac{1}{2} x^{2}}$.

Secondly each block is then locally evaluated to capture the temporal evolution of the energy ratio.

When an energy drop is observed for a significant period of time an edge smoothing process occurs. The edge smoothing process can be affected by noise due to light variation in the scene. A Mixture of Gaussian model is adopted to improve the analysis robustness.

The MoG has the great advantage to correctly catch variations for multimodal distributions. To compute the probability for each frame the on-line expectation maximization algorithm proposed in [11] is used.

In detail, for all blocks $b_{k}$ of the image $I_{t}$ at time $t$ the value $r\left(b_{k}, I_{t}, B g_{t}\right)$ is computed and the MoG of block $b_{k}$ updated using a selective update method. This process have a main advantage. The mixture component reweighting process is able to catch slow and gradual variations of energy ratio. Values that do not occur frequently are filter out and assigned to the least probable Gaussian of the mixture. This property is helpful for evaluating the gradient intensity lowering process of smoking regions that has the peculiarity of being slow and continuous in time, Fig. 3.

To capture the time variation of the energy ratio the Gaussian Mixture Model 

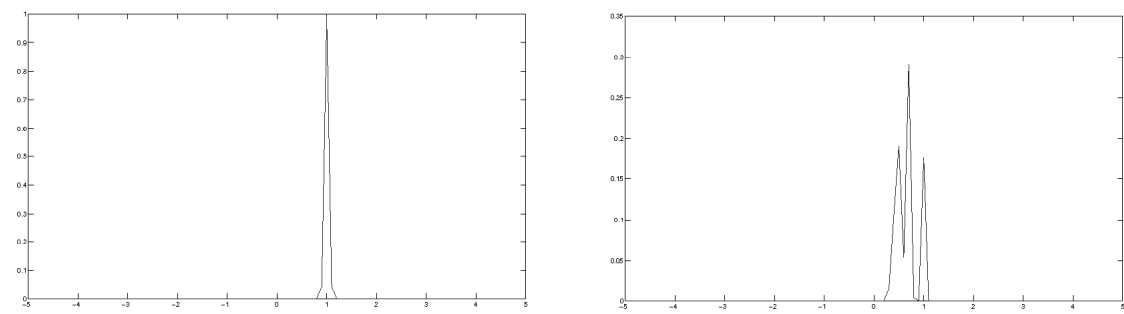

Fig. 3. Gaussian mixtures obtained observing energy ratio values at a single block. The left plot shows the mixture when there is no smoke in the block. The right plot shows how the mixture changes when smoke is in the scene. It is worth noting that when a block is covered by smoke the mixture components mean values move gradually towards 0 .

was preferred to a Hidden Markov model (HMM). Although HMMs are widely adopted to classify and model temporal stochastic processes, the data values sequence is crucial to obtain a good classification. Instead, as previously stated, the block energy ratio is subject to strong fluctuations of energy values due to noise and natural scene lighting. This reason makes the lowering sequence unpredictably variable in different setups; thus the specific energy drop trajectory can produce misleading results. On the contrary is interesting to analyze the global trend.

\subsection{Color analysis to detect blended smoke regions}

When a smoke event occurs, scene regions covered by smoke change their color properties. The smoke can either be completely opaque or partially transparent. In the former case the covered region changes completely its color while in the latter case the color of the covered region appears to be blended with the smoke color.

This simple observation remains valid in all the observed cases and intuitively suggests a hint to characterize the color of a smoke region.

The proposed model simply adopts an evaluation based on a blending function mutuated from computer graphics. A reference color model is chosen in the RGB color space to represent the color of the smoke in the scene. The model is selected by analyzing the different color tones produced combusting different materials. For explanatory purposes is possible to concentrate the analysis to the case of a light gray color model as the smoke in the leftmost image of Fig. 1. Each pixel $I_{t}(i, j)$ of the input frame at time $t$ is then checked against the smoke model and the background model $B g_{t}$ to evaluate the reference color presence computing the blending parameter $b l$ using equation 4 . The evaluation takes into account 
the case where the scene color and the smoke color are mixed together.

$$
b l\left(i, j, I_{t}, B g_{t}, S\right)=\frac{I_{t}(i, j)-B g_{t}(i, j)}{S-B g_{t}(i, j)}
$$

where $B g_{t}$ is the current backround model at time $t$ and $S$ is the smoke reference color model.

To filter out the errors and possible measurements inaccuracy the blending value is computed for each image block as the average of $b l$ values in the block:

$$
\beta_{b_{k}}\left(I_{t}, B g_{t}, S\right)=\frac{1}{N^{2}} \sum_{i, j \in b_{k}} \frac{I_{t}(i, j)-B g_{t}(i, j)}{S-B g_{t}(i, j)}
$$

where block size is $N \times N$

In conclusion the $\beta$ measure quantifies how much each block globally shares chromatic properties with the reference color model.

\subsection{A Bayesian approach for classification}

In the previous subsections the blockwise energy ratio measure $r$ and the color blending measure $\beta$ have been presented as possible discriminant features to identify a smoke region in the scene. A Bayesian formulation has been chosen to identify whether a block $b_{k}$ is likely to belong to a smoke region. For each block the posterior probability of smoke presence, the event $f=1$, considering the block $b_{k}$ is defined:

$$
P\left(f=1 \mid b_{k}\right) \propto P\left(b_{k} \mid f=1\right) P(f=1)
$$

The likelihood value is obtained by combining both the contributions coming from energy ratio and color information. These terms are considered probabilistically independent to simplify the treatment.

$$
P\left(b_{k} \mid f=1\right)=P\left(r_{b_{k}}, \beta_{b_{k}} \mid f=1\right)=P_{r}\left(b_{k} \mid f=1\right) \cdot P_{\beta}\left(b_{k} \mid f=1\right)
$$

The likelihood contribution due to energy ratio decay is obtained by summing the weighted Gaussians of the MOG having mean value below a considered threshold $t h_{1}$ computed empirically observing the mean energy ratio value in smoke regions.

$$
P_{r}\left(b_{k} \mid f=1\right)=\sum_{i=1}^{K} w_{i} N\left(r\left(b_{k}, I_{t}, B g_{t}\right) \mid \mu_{i} \sigma_{i}\right)
$$

when the $i^{\text {th }}$ Gaussian mean value $\mu_{i}<t h_{1}$.

The color contribution to the likelihood value is directly computed as the block color blending measure $\beta_{b_{k}}$ according to equation 5 .

$$
P_{\beta}\left(b_{k} \mid f=1\right)=B_{k}\left(I_{t}, B g_{t}, S\right)
$$


The classification is biased making use of prior knowledge acquired observing several clips containing smoke. The prior probability of a smoke event in the current frame is directly related to the mean energy ratio value of the scene and computed using the non parametric distribution obtained by equation 3 .

$$
P(f=1)=\widehat{f}\left(\frac{1}{M} \sum_{\forall b_{k} \in I_{t}} r\left(b_{k}, I_{t}, B g_{t}\right)\right)
$$

where $I_{t}$ is composed by $M$ blocks.

The posterior probability value is thresholded to identify a candidate smoke block. The test for smoke presence is performed after foreground object segmentation. For any segmented object in the scene the number of candidate blocks intersecting the object's blob is computed. Finally an object is classified as smoke when the $70 \%$ of its area overlays candidated smoke blocks.

\section{Experimental results and discussion}

The proposed smoke detection system can be used in conjunction with a whichever video surveillance system providing moving object segmentation using a background model. The background model should be updated regularly but smoke regions should not be included in the background. This can be achieved choosing a slow background update rate and avoiding updating the background model areas where a smoke object is detected. The tests were performed using both the Stauffer and Grimson background model with selective update [4] and the SAKBOT median background model with knowledge based update [9]. Although the results did not vary significantly changing the background model and object detection technique, the second method has been preferred since discriminates the presence of possible shadows objects too.

\begin{tabular}{|l|c|l|c|c|c|c|c|c|}
\hline \multicolumn{2}{|c|}{} & \multicolumn{2}{|c|}{ Temporal Analysis } & \multicolumn{2}{c|}{ Color Analysis } & \multicolumn{2}{c|}{ Gloabal Analysis } \\
\hline Morie Name & Frame Number & Outdoor/ndoor & Time To Detect & False positive & Time To Detect & False positive & Time To Detect & False positive \\
\hline movie_01 & 165 & Outdoor & 22 & - & 1 & - & 1 & - \\
movie_02 & 210 & Indoor & 18 & - & 1 & - & 1 & - \\
movie_03 & 2200 & Outdoor & 28 & - & 34 & - & 20 & - \\
movie_04 & 3005 & Indoor & 212 & - & 273 & - & 185 & - \\
movie_05 & 1835 & Indoor & 87 & - & 100 & 3 & 52 & - \\
movie_06 & 2345 & Outdoor & 129 & - & 161 & - & 116 & - \\
movie_07 & 2024 & Indoor & 57 & 3 & 99 & - & 35 & - \\
movie_08 & 2151 & Outdoor & 88 & 2 & 88 & - & 42 & - \\
movie_09 & 1880 & Outdoor & 59 & - & 56 & - & 45 & - \\
movie_10 & 2953 & Outdoor & 457 & - & 498 & - & 300 & - \\
movie_11 & 1485 & Indoor & 62 & - & $x$ & 5 & 62 & - \\
movie_12 & 499 & Outdoor & 43 & - & 8 & - & 16 & - \\
movie_13 & 195 & Indoor & 53 & - & 23 & - & 27 & - \\
movie_14 & 1226 & Outdoor & 77 & - & 370 & - & 69 & - \\
movie_15 & 109 & Outdoor & 29 & - & $x$ & 1 & 3 & - \\
\hline
\end{tabular}

Fig. 4. Experimental results of the proposed system on reference clips. 
In all the tests carried out the learning rate $\alpha$, [4], of the MoGs used to model the energy ratio decay was set to 0.1 . Although changing this parameter does not have major effects on the system performance, a fast learning rate is preferable to detect energy ratio variations rapidly. The system was tested on 50 clips of varying length in both indoor and outdoor setups where moving objects such as people or vehicles were present in the scene during the smoke event. Each clip contained a smoke event. Part of the dataset is publicly available at website http://imagelab.ing.unimore.it/visor. Each likelihood term was evaluated separately to measure the impact on the system performance.

The table in Fig. 4 summarizes the results obtained on 15 reference clips.

The first column of the table reports the video type and its frame-length. The average clips framerate is $25 \mathrm{fps}$. The remaining columns report the results obtained using each likelihood term separately and finally the results of the whole system. The detection time after the smoke event occurs is reported for all the test clips. The table clearly shows that the likelihood term due to temporal analysis (eq.8) is effective in most of the observed cases. The main problem is the long detection time. This is caused by the time based statistics used to capture the energy ratio decay. Although the likelihood contribution due to color blending has the advantage of speed up the detection process it tends to detect much false positives if used alone. See seventh column of Fig. 4. Observing the last two columns of Fig. 4 we can state that the complete approach is fast and reliable enough even in situations where each likelihood contribution fails. The overall system results on the 50 clips used for testing purposes report a detection rate of $77 \% 3$ seconds later the smoke event occurs, $98.5 \% 6$ seconds later and finally $100 \% 10$ seconds later with an average true positive rate of 4\%. Fig. 5 shows some snapshots of the system working on different conditions.

\section{Conclusions and acknowledgments}

In conclusion we propose a system capable of detecting if foreground objects are smoke or not using both wavelet transform energy coefficients and image color properties. The proposed Bayesian approach has been extensively evaluated on public data and results in term of detection rate and time to detect have been reported. The adoption of a two-contribution likelihood measure solves most of the emerged problems of each chosen feature and boosts up significantly the detection process. The system well performed in all the tested scenarios and results are very robust.

This work is partially supported by the project BESAFE (Behavior lEarning in Surveilled Areas with Feature Extraction) funded by NATO Science for Peace programme and by the project FREE SURF funded by Italian MIUR Ministry.

\section{References}

1. Kopilovic, I., Vagvolgyi, B., Sziranyi, T.: Application of panoramic annular lens for motion analysis tasks: surveillance and smoke detection. In:Proceedings of 15th 

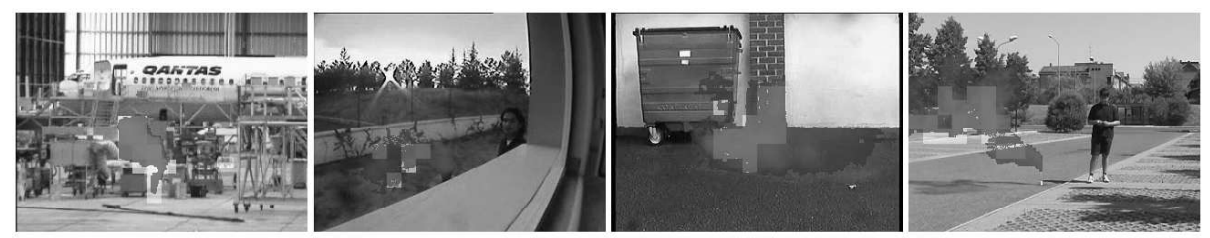

Fig. 5. Snapshots of the proposed system working on several clips in different conditions. The blue area in the images is detected as smoke.

International Conference on Pattern Recognition Vol. 4. IEEE, 3-7 Sept. (2000)714717

2. Vicente, J., Guillemant, P.: An image processing technique for automatically detecting forest fire. In:International Journal of Thermal Sciences, Vol. 41, Issue 12 (2002) 1113-1120.

3. Thou-Ho (Chao-Ho) Chen, Yen-Hui Yin, Shi-Feng Huang, Yan-Ting Ye: The Smoke Detection for Early Fire-Alarming System Base on Video Processing. In: International Conference on Intelligent Information Hiding and Multimedia (2006) 427-430.

4. Stauffer, C., Grimson, W.E.L.: Adaptive Background Mixture Models for RealTime Tracking. In:Proceedings IEEE Conference on Computer Vision and Pattern Recognition. IEEE (1999) 246-252.

5. Xiong Z., Caballero R., Wang H., Finn A., Lelic M. A., Peng P.: Video-based Smoke Detection: Possibilities, Techniques, and Challenges Suppression and Detection Research and Applications. In:A Technical Working Conference (SUPDET 2007). March 5-8 (2007) Orlando, Florida.

6. Toreyin, B.U., Dedeoglu, Y., Cetin A.E.: Flame detection in video using hidden Markov models. In:IEEE International Conference on Image Processing IEEE (2005).

7. Toreyin, B.U., Dedeoglu, Y., Cetin, A.E., Fazekas, D., Chetverikov, T. Amiaz, N. Kiryati.: Dynamic texture detection, segmentation and analysis In:Conference On Image And Video Retrieval. ACM (2007) 131-134.

8. Collins, R. T., Collins, A. J., Lipton, Kanade, T.: A system for video surveillance and monitoring In: 8th International Topical Meeting on Robotics and Remote Systems. American Nuclear Society (1999).

9. R. Cucchiara, R., Grana, C., Piccardi, M., Prati, A. Detecting Moving Objects, Ghosts and Shadows in Video Streams In: IEEE Transactions on Pattern Analysis and Machine Intelligence. IEEE Vol. 25, Issue 10 (2003) 1337-1342.

10. Mallat, S. G., A theory for multiresolution signal decomposition: The wavelet representation In: IEEE Transactions on Pattern Recognition and Machine Intelligence IEEE Vol. 11, Issue 7 (1989) 674-693.

11. Sato, M., Fast learning of on-line EM algorithm. In:Technical Report TR-H-281, ATR Human Information Processing Research Laboratories. 